«Системні технологіï» 1 (126) 2020 «System technologies»

DOI 10.34185/1562-9945-1-126-2020-01

УДК 004-93

Л.Г. Ахметшина, С.В. Мазурик, И.А. Скуратовский

\title{
ПРОГНОЗИРОВАНИЕ СВОЙСТВ МНОГОКОМПОНЕНТНОЙ КЕРАМИКИ НА ОСНОВЕ САМООРГАНИЗУЮЩЕЙСЯ СЕТИ КОХОНЕНА
}

Аннотация. Рассмотрены информационные возможности метода прогнозирования значений многомерных экспериментальных данных, определенных на неравномерной сетке. Суть метода заключается в использовании самоорганизующейся сети Кохонена для определения качественного влияния независимых параметров в многокомпонентной керамике на основе оксида олова на коэффициент ее нелинейности в задаче улучшения параметров элементов защиты электрических цепей.

Ключевые слова: самоорганизующаяся сеть Кохонена, многокомпонентная керамика, прогнозирование свойств, кластеризация, визуализация.

Введение. Существует множество прикладных задач, требующих применения методов прогнозирования данных, например, при оценке свойств многокомпонентных материалов. В частности, керамические материалы формируются на основе нескольких исходных компонентов и определение их требуемых составов является трудоемким процессом, поскольку целевые свойства могут существенно изменяются при незначительном варьировании любой из независимых компонент. Задача построения физической модели усложняется из-за нелинейного поведения керамических материалов в зависимости от процентного содержания составляющих, низкой воспроизводимости результатов физических экспериментов, невозможности описания одновременного взаимодействия многокомпонентных материалов и сложностью графического отображения зависимости их целевых свойств.

Увеличение количества проведенных экспериментов повышает точность прогноза, однако, во многих практических случаях данные измерений имеют незначительный объем, распределены нерегулярным

(C) Ахметшина Л.Г., Мазурик С.В., Скуратовский И.А., 2020 


\section{«Системні технології» 1 (126) 2020 «System technologies»}

образом и отсутствует возможность проведения дополнительных экспериментов из-за дорогостоящих лабораторных исследований.

В настоящее время альтернативой физическим моделям является подход, основанный на методах искусственного интеллекта, в частности, использования самоорганизующихся нейронных сетей Кохонена, которые делают возможным выполнение прогноза на основе небольших объемов информации [1]. Они хорошо зарекомендовали себя при моделировании систем и процессов, внутренние ограничения в которых либо недостаточно изучены, либо взаимодействуют сложным образом [2].

Анализ публикаций. Самоорганизующаяся карта (SOM) или нейронная сеть Кохонена является одним из основных типов искусственных нейронных сетей с конкурентоспособным обучением без учителя, в которой используется набор классификационных нейронов, которые самоорганизуются в пространстве на основе только имеющихся входных данных.Обучение SOM - это отображение многомерного пространства на двумерную сетку нейронов. Проецирование входного пространства большого размера на топологию низкого размера позволяет обнаруживать связи между сложными данными и широко используется в последние годы для решения различных задач [3]. Применение стандартного SOM, представленного Кохоненом, и его вариантов для прогнозирования изучалось в литературе при различных условиях, в частности, для временных рядов [2] и интерполяции пространственных данных[4].

Постановка задачи. В данной работе исследуется информационные возможности применения самоорганизующихся карт Кохонена для оценки качественного влияния составляющих многокомпонентной варисторной керамики на основе оксида олова на ее свойства на примере прогнозирования коэффициента нелинейности.

Основная часть. Традиционный путь получения керамики из оксидов металлов заключается в смешивании в некоторых пропорциях исходных составляющих и получение шликера с применением органических связующих. Из шликера формируются заготовки нужной формы, которые подвергаются обжигу с заданными параметрами нагрева. Каждый из оксидов металлов вносит вклад в результирующие свойства ком4 
«Системні технологіï» 1 (126) 2020 «System technologies»

позита, причем их влияние определяется опытным путем на основе изучения графиков вольт-амперных характеристик (BAX) образцов керамики без соответствующего оксида металла и с его различным содержанием [5].

На рис. 1 приведены графики ВАХ 4-х компонентной керамики $\mathrm{ZnO}-\mathrm{PbO}-\mathrm{Co}_{3} \mathrm{O}_{4}-\mathrm{B}_{2} \mathrm{O}_{3}$ (оксиды цинка, свинца, кобальта и бора). Кривая 1 рис. 1 а соответствует полному составу, который характеризуется высоким значением коэффициента нелинейности $\beta \approx 40$. Анализ графиков BAX рис. 1 а, при отсутствии одного из компонентов, позволяет сделать такие выводы о влиянии составляющих на изменение свойств смеси:

- $\mathrm{Co}_{3} \mathrm{O}_{4}$ (кривая 2) - наклон ВАХ и, соответственно, коэффициент нелинейности уменьшаются;

$-\mathrm{B}_{2} \mathrm{O}_{3}$ (кривая 3), $\mathrm{PbO}$ (кривая 4) - ВАХ линейна $(\beta \approx 1)$, при этом образец имеет высокую электропроводимость, что свидетельствует о том, что не происходит образование потенциальных барьеров на границах зерен и проводимость увеличивается.

Более сложным является поведение BAX образцов керамики с изменением содержания оксида свинца (рис. 1 б). При увеличении содержания $\mathrm{PbO}$ от нуля до 4\% BAX смещается в сторону роста величины электрического поля и при этом растет коэффициент нелинейности (кривые 1-3 рис. 1 б). Увеличение содержания оксида свинца в большей степени ограничивает рост зерен ZnO и способствует образованию потенциальных барьеров на все большем числе границ зерен. Дальнейший рост концентрации $\mathrm{PbO}$ вызывает повышение коэффициента нелинейности до значений 45-52 (рис. 1 - кривые 4, 5), но сдвиг ВАХ прекращается. Это происходит из-за накопления фазы на основе $\mathrm{PbO}$ в местах контактов нескольких зерен. Увеличение примеси $\mathrm{PbO}$ до $12 \%$ приводит к уменьшению коэффициента нелинейности до 11 и снижению напряженности поля при фиксированном значении плотности тока (кривая 6 рис. 1 б).

В случае накопления фазы на основе $\mathrm{PbO}$ в местах контактов нескольких зерен (при большом содержании $\mathrm{PbO}$ в обжигаемом образце) эта фаза может производить шунтирующее действие для потенциально- 
«Системні технології» 1 (126) 2020 «System technologies»

го барьера на границах зерен, тем самым снижая коэффициент нелинейности и смещая BAX в область несколько меньших значений электрического поля (рис. 1 б, кривые 4-6).

Таким образом, пример показывает, что при исследовании многокомпонентных образцов керамики необходим большой объем экспериментальной работы, которая усложняется отсутствием априорной информации о необходимом процентном составе исходных составляющих.

$\lg 10^{4}, \mathrm{~A} / \mathrm{m}^{2}$

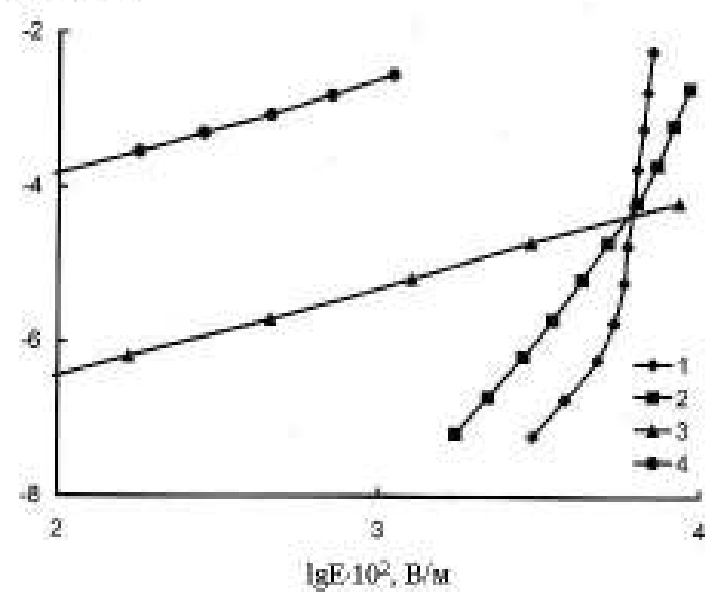

a

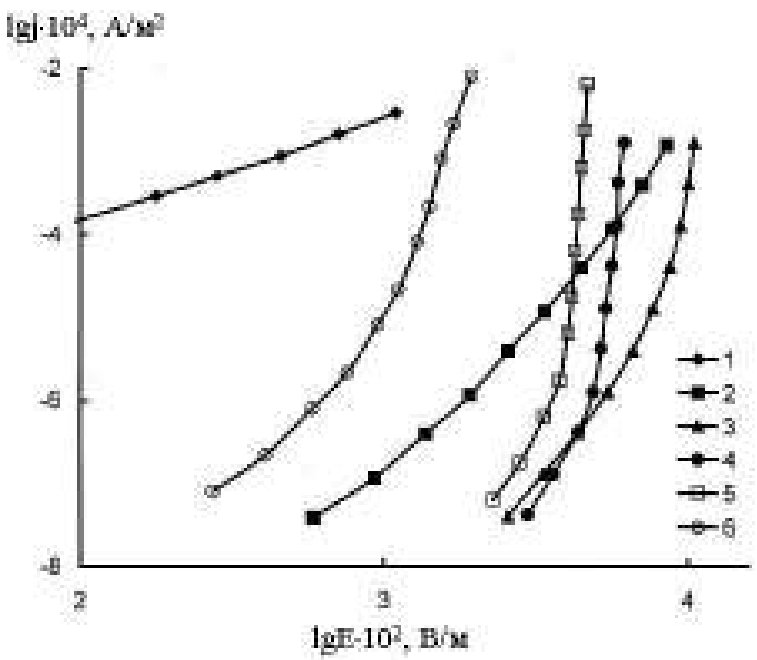

6

Рисунок 1 - Вольт-амперные характеристики варисторной керамики системы $\mathrm{ZnO}-\mathrm{PbO}-\mathrm{Co}_{3} \mathrm{O}_{4}-\mathrm{B}_{2} \mathrm{O}_{3}$ :

a) 1 - $\mathrm{ZnO}-\mathrm{PbO}-\mathrm{Co}_{3} \mathrm{O}_{4}-\mathrm{B}_{2} \mathrm{O}_{3}$ и без одного компонента:

2- $\mathrm{ZnO}-\mathrm{PbO}-\mathrm{B}_{2} \mathrm{O}_{3} ; 3-\mathrm{ZnO}-\mathrm{PbO}-\mathrm{Co}_{3} \mathrm{O}_{4} ; 4-\mathrm{ZnO}^{-} \mathrm{Co}_{3} \mathrm{O}_{4}-\mathrm{B}_{2} \mathrm{O}_{3}$;

б) с изменением содержания $\mathrm{PbO}: 1-0 ; 2-1,5 \% ; 3-4,5 \% ; 4-7,7 \%$;

$$
5-9 \% ; 6-12 \%
$$

В данной работе применена нейронная сеть SOM для прогнозирования свойств многокомпонентной керамики на основе оксида олова по данным физических экспериментов. При использовании метода «интеллектуальной» аппроксимации с использованием SOM по завершении процесса сходимости алгоритм отображает важные статистические характеристики входного пространства, вид функциональной зависимости определяется автоматически.

Нейроны сети образуют двумерную решетку и связаны со всеми узлами входного слоя: $n_{x}, n_{y}$ - число узлов в карте по соответствующим координатным осям; $\left(x_{1}, x_{1}, \ldots x_{n}\right)$ - исходные данные размерности $n=7$; 6 
«Системні технологіï» 1 (126) 2020 «System technologies»

$W_{i}=\left[w_{i l}, w_{i 1}, \ldots w_{i 1},\right]^{T}$ весовые коэффициенты $i$-го узла. Задача состоит в том, чтобы аппроксимировать данные, $k k=n_{x} \mathrm{x} n_{y}$ узлам сети. На результаты прогноза существенное влияние оказывает как общее число нейронов в сети, так и соотношение количества нейронов $n_{x}, n_{y}$ по осям $X$, Y.C каждым нейроном связывается модельный вектор, т.е. координаты узла в пространстве сигналов представляются в виде соответствующего нейрону множества вещественных чисел. Если количество нейронов избыточно, появляются «пустые» узлы. Нейронная сеть позволяет получить проекцию всего пространства наблюдений, т. е. различные нейроны оказываются чувствительными к различным областям данных, сегментация осуществляется адаптивно.

Поиск «нейрона-победителя» (ближайшего узла) $m_{c}$ производится с использованием критерия подобия. Для евклидова расстояния, используется формула $\arg \left(\min \left\|x-w_{j}\right\|\right), j=1, \ldots k$. Коррекция синаптических весов нейронов-соседей выполняется на основе выражения $\left.w_{j}(n+1)=w_{j}(n)+\mu(n) h_{i j}(n)\left(x-w_{j}(n)\right)\right)$, а для остальных узлов $w_{j}(n+1)=w_{j}(n)$, где $n$ - номер шага; $0<\mu(n)<1$ - параметр скорости обучения, убывающий с каждым шагом; $h_{i j}(x)$ - функция окрестности (соседства) с центром победившего нейрона, определяющая силу взаимного влияния любой пары узлов сети - расстояние на которое передается коррекция. Эти параметры динамически изменяются во время обучения с целью получения лучшего результата.

После обучения сети, целевое значение, которое подлежит восстановлению, ассоциируется с ближайшим узлом сетки, что означает замену точки на центр локального сгущения данных в пространстве, которое хранится в кодовом блокноте (codebook).

Следует отметить, что важным элементом технологии нейросетевого анализа является предобработка данных, которая может включать масштабирование, нормализацию, удаление регулярностей и т.д., что повышает скорость и качество обучения $[1,2]$.

Экспериментальные результаты. В качестве исходных данных для SOM использовались результаты исследования варисторной керамики на основе оксида олова $\mathrm{SnO}_{2}$, содержание которого было не меньше 
«Системні технології» 1 (126) 2020 «System technologies»

95\% [6]. Для оценки информативных возможностей метода были составлены и изготовлены 42 образца пятикомпонентной смеси с примесями $\mathrm{Cr}_{2} \mathrm{O}_{3}, \mathrm{~B}_{2} \mathrm{O}_{3}, \mathrm{Sb}_{2} \mathrm{O}_{5}, \mathrm{Co}_{3} \mathrm{O}_{4}$, значения которых изменялись в диапазоне 0,04 до 5\%. Образцы получены по стандартной технологии с температурой обжига 1100С, выдержкой в течении часа при максимальной температуре и одинаковыми скоростями нагрева и охлаждения печи для обжига.

Целью анализа экспериментальных данных является определение влияния состава примесей на значение коэффициента нелинейности alpha варисторной керамики: необходимо получить возможно большую величину alpha при низкой напряженности электрического поля $\boldsymbol{E}$ (параметры измерялись при одинаковой плотности тока).

Отличительной особенностью входных данных является малый объем выборки, неравномерный характер изменения свойств, непрезентативный набор и существенное различие в диапазоне изменения независимых и целевых атрибутов. Для повышения скорости и качества обучения было выполнено приведение исходных значений к единому масштабу за счет нормировки по диапазону.

Проверка прогнозирующих свойств карты и определение оптимальных параметров проводилась в ходе обучения на 39 входных образцах, остальные использовались в качестве тестовых. При выполнении эксперимента использовались следующие значения параметров алгоритма: $n_{x}=70 ; n_{y}=70 ; h_{i j}(x)$ - гауссова функция модификации соседних нейронов; изменение радиуса влияния нейрона для грубой фазы определяется как целая часть от значения $r_{1}=\left(n^{2}+n_{y}\right)^{1 / 2}$, а для точной фазы как $r_{2}=$ $r_{1} / 5$. Значение радиуса соседства оказывает критическое влияние на точность результата.

На рис. 2 представлена полученная структура карт признаков, анализ которых показывает, что максимальное значение целевой переменной alpha совпадает с областью незначительной величины $\boldsymbol{E}$, а компонента $\mathrm{Co}_{3} \mathrm{O}_{4}$ практически не оказывает влияния в области интереса это допускает возможность сокращения пространства анализируемых признаков. Для оценочного моделирования вполне достаточна непосредственная визуализация кодового блокнота (codebook).Ha рис. 3 а 8 
«Системні технології» 1 (126) 2020 «System technologies»

приведена поверхность целевого параметра alpha с нанесенными значениями исходных данных.

Проверка прогнозирующих свойств проводилась по двум направлениям - восстановление null-значений отдельных параметров, включая целевое, и прогнозирование новых шаблонов на основе значений центроидов кластеров, соответствующих ближайшему к целевому прототипу.

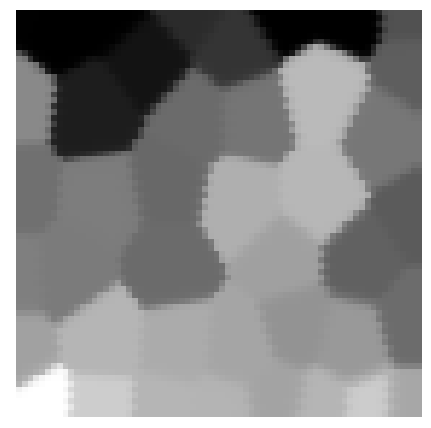

Alpha

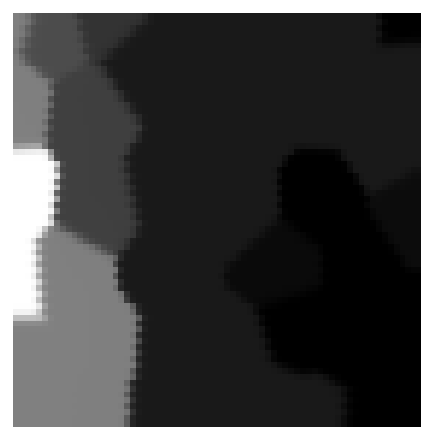

$\mathrm{B}_{2} \mathrm{O}_{3}$

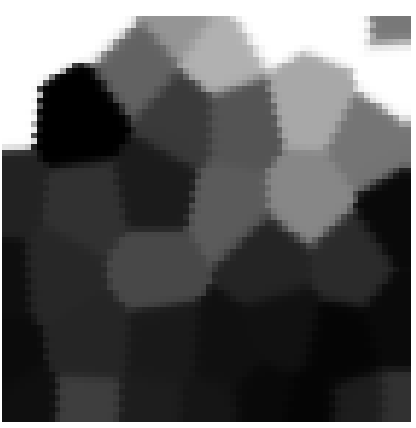

$\boldsymbol{E}$

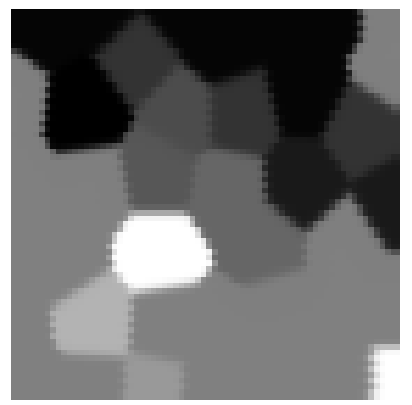

$\mathrm{Sb}_{2} \mathrm{O}$

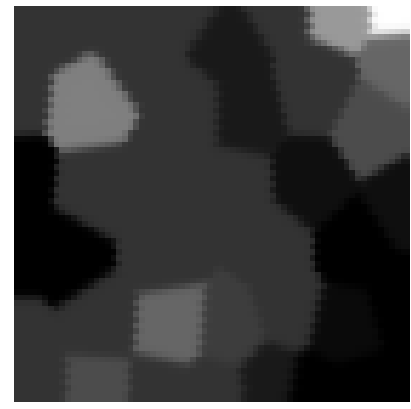

$\mathrm{Cr}_{2} \mathrm{O}_{3}$

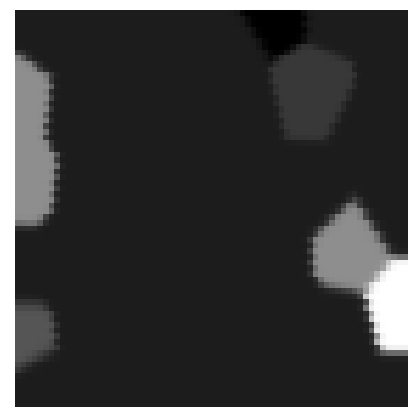

$\mathrm{Co}_{3} \mathrm{O}_{4}$

Рисунок 2 - Карты признаков для сети с nx=70; ny=70

На рис. $3 б$ приведена поверхность одной из независимой переменных с тестовыми точками, значение которых прогнозировались в ходе эксперимента. 


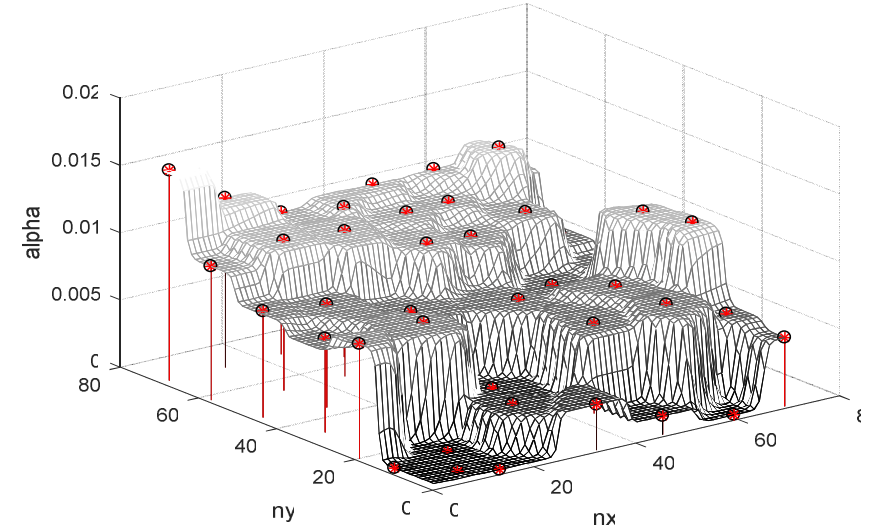

a

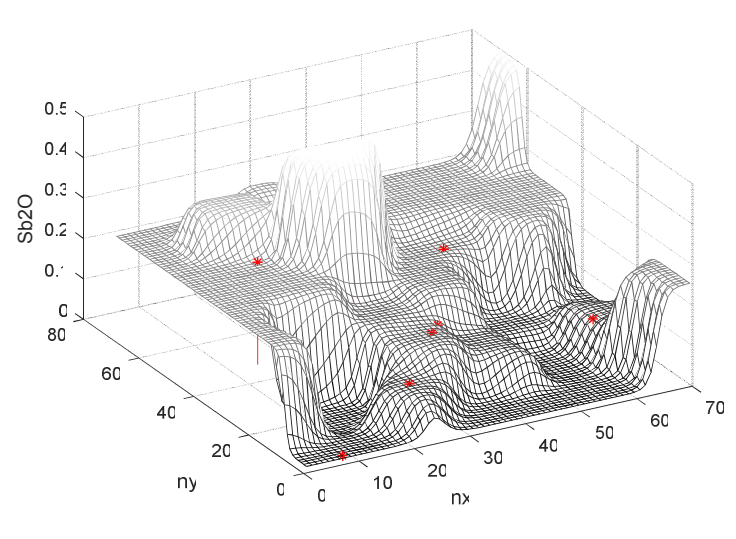

6

Рисунок 3 - Поверхность SOM: а-коэффициент нелинейности с экспериментальными точками; б- $\mathrm{Sb}_{2} \mathrm{O}$ с данными прогноза

Совместная 2D и 3D-визуализация различных составляющих карты позволяет выявить наиболее перспективные области входных данных для проведения дальнейших физических экспериментов.

\section{Выводы:}

- на основе интеллектуального прогнозирования с использованием SOM определены области перспективных изменений перспективные значения состава керамики для дальнейших физических экспериментов;

- использование SOM обеспечивает реализацию достаточно универсальной технологии получения качественной оценки зависимости целевого значения от независимых составляющих для ограниченного набора экспериментальных данных;

- повышение эффективности анализа данных при низкой изменчивости и ограниченном количестве экспериментов может быть достигнуто при использовании нечеткого подхода.

\section{ЛИТЕРАТУРА / ЛІТЕРАТУРА}

1. Кохонен Т. Самоорганизующиеся карты /Кохонен Т. [пер. 3-го англ. изд. В.Н. Агеева под ред. Ю.В.Тюменцева]. -М.:Бином. Лаборатория знаний, 2008. -665 с.

2. Егоров А. Оптимизация яркости изображений на основе нейро-фаззи технологий / А. Егоров, Л. Ахметшина// Монография.- Изд. Lambert. $-2015 .-139$ c.

3. Yang H. Self-organizing nonlinear output (SONO): a neural network suit- 
«Системні технології» 1 (126) 2020 «System technologies»

able for cloud patch-based rainfall estimation from satellite imagery at small scales / H. Yang, K. L. Hsu, S. Sorooshian, and X. Gao // Water Resources Research.-vol. 41.-Article ID W03008.-2005.

4. Ахметшина Л. Г. Метод интерполяции неравномерных пространственных данных на основе сети Кохонена/Л. Г. Ахметшина, А. М. Ахметшин, И. М. Удовик // Системні технології.-2013.-Дніпропетровськ.-№ 1. - С. 106-112.

5. Глот А.Б. Керамика $\mathrm{ZnO}-\mathrm{PbO}-\mathrm{Co}_{3} \mathrm{O}_{4}-\mathrm{B}_{2} \mathrm{O}_{3}$ с неомической электропроводностью / А.Б.Глот, С.В.Мазурик // Неорганические материалы.- 1998.T. 34.- № 2.- C. 247-250.

6. Glot A. B. Non-Ohmic conduction in tin dioxide based varistor ceramics/ Glot A. B., Skuratovsky I. A.// Materials Chemistry and Physics.-2006.- Vol. 99, № 6.- P. 487-493.

\section{REFERENCES}

1. Kokhonen T. Samoorhanizuyutsya karty / Kokhonen T. [per. 3-ho anhl. yzd. V.N. Ahyeyevapid red. YU.V. Tyumentseva]//.M .: Binom. Laboratoriya znan.2008. - $665 \mathrm{~s}$.

2. Yehorov A. Optymizatsiya yaskravosti zobrazhen naosnovi neyrofazzi tekhnolohiy / A. Yehorov, L. Akhmetshyna// Monohrafiya.- Vyd. Lambert. 2015. -139 c.

3. Yang H. Self-organizing nonlinear output (SONO): a neural network suitable for cloud patch-based rainfall estimation from satellite imagery at small scales/ H. Yang, K. L. Hsu, S. Sorooshian, and X. Gao // Water Resources Research.- vol. 41.- Article ID W03008.- 2005.

4. L.G.Akhmetshina. Metod interpolyatsii neravnomernykh prostranstvennykh dannykh na osnove seti Kokhonena / L. G.Akhmetshina, A. M. Akhmetshin, I. M. Udovik // Sistemní tekhnologíí, -2013. -Dnípropetrovs'k.- № 1. - S. 106112.

5. Glot A.B. Keramika ZnO-PbO- $\mathrm{Co}_{3} \mathrm{O}_{4}-\mathrm{B}_{2} \mathrm{O}_{3} \mathrm{~S}$ neomicheskoj elektroprovodnostyu/ A.B. Glot, S.V. Mazurik// Neorganicheskie materialy, 1998.- T. 34.- № 2.- S. 247-250.

6. Glot A. B. Non-Ohmic conduction in tin dioxide based varistor ceramics/ Glot A. B., Skuratovsky I. A.// Materials Chemistry and Physics.- 2006.- Vol. 99, № 6.- P. 487-493. 


\section{«Системні технології» 1 (126) 2020 «System technologies»}

\section{Прогнозування властивостей багатокомпонентних керамік на основі мережі Кохонена, що самоорганізується}

Розглянуті можливості застосовування нейронної мережі Кохонена для оцінки якісного впливу, складових багатокомпонентної кераміки на їі властивості. Представлені експериментальні результати аналізу експериментальних даних щодо застосування прогнозованих коефіцієнтів нелінійності п'ятикомпонентної суміші варисторної кераміки на основі оксиду олова із застосуванням Cr203, B203, Sb205, Co304.

\section{Prediction of the properties of multicomponent ceramics based on the Kohonen self-organizing network}

The information possibilities of the method for predicting the values of multidimensional experimental data determined on an uneven grid are considered. The essence of the method is to use the Kohonen self-organizing network to determine the qualitative effect of independent parameters in tin oxide-based multicomponent ceramics on its non-linearity coefficient in the task of improving the parameters of electrical circuit protection elements.

There are many applications that require the application of data forecasting methods, for example, when evaluating the properties of multicomponent materials. In particular, ceramic materials are formed on the basis of several initial components and the determination of their required compositions is a laborious process, since the target properties can significantly change with a slight variation of any of the independent components. Thus, the study of multicomponent ceramic samples requires a large amount of experimental work.

Currently, an alternative is an approach based on artificial intelligence methods, in particular, the use of Kohonen's self-organizing neural networks, which make it possible to make forecasts based on small amounts of information. The purpose of the analysis of experimental data is to determine the influence of the composition of impurities on the nonlinearity coefficient alpha of varistor ceramics: it is necessary to obtain the largest possible value of alpha at a low electric field strength $E$ (parameters were measured at the same current density). Verification of the predictive properties was carried out in two directions - restoration of the null-values of individual parameters, including the target and forecasting of new patterns based on the values of the centroids of the clusters corresponding to the closest to the target prototype.

On the basis of intelligent forecasting using SOM, the areas of promising changes are determined for the future values of the composition of ceramics for further physical experiments. Using SOM provides the implementation of a fairly universal technology for obtaining a qualitative assessment of the dependence of the target value on independent components for a limited set of experimental data. 
«Системні технології» 1 (126) 2020 «System technologies»

Ахметшина Людмила Георгиевна - профессор, д.т.н., профессор кафедры эл-тронных вычислительных машин Днепровского национального университета имени Олеся Гончара.

Мазурик Станислав Васильевич - ассистент кафедры электронных выч-слювальних машин, Днепровский национальный университет имени Олеся Гончара.

Скуратовский Игорь Анатольович - кандидат физико-математических наук, доцент, доцент кафедры радиоэлектроники, Днепровский национальный университет имени Олеся Гончара.

Ахметшина Людмила Георгіївна - професор, д.т.н., професор кафедри електронних обчислювальних машин Дніпровського національного університету імені Олеся Гончара.

Мазурик Станіслав Васильович - асистент кафедри електронних обчислювальних машин, Дніпровський національний університет імені Олеся Гончара.

Скуратовський ггор Анатолійович -кандидат фізико-математичних наук, доцент, доцент кафедри радіоелектроніки, Дніпровський національний університет імені Олеся Гончара.

Akhmetshina Lyudmila - Doctor of Technical Sciences, Professor, Professor of the Department of Electronic Computing Machinery of the Oles Honchar Dnipro National University.

Mazurik Stanislav - assistant of the department of electronic computers of the faculty of physics electronics and computer systems of the Oles Honchar Dnipro National University.

Skuratovsky Ihor-- associate professor, candidate of physical and mathematical sciences, associate professor at the Department of Radio Electronics of Oles Honchar Dnipro National University. 